\title{
Räumliche Regressionsmodelle als leistungsfähige Methoden zur Erklärung der Driving Forces von Zuzügen in der Stadtregion Wien?
}

\author{
Marco Helbich • Peter J. Görgl
}

Eingegangen: 4. Juni 2009 / Angenommen: 9. November 2009 / Online publiziert: 17. Februar 2010

(C) Springer-Verlag 2010

\begin{abstract}
Zusammenfassung Die Bevölkerungsentwicklung in Großstadtregionen ist ein kontinuierlicher dynamischer Prozess, der auf unterschiedliche Art und Weise analysiert werden kann. Dieser Beitrag stellt zwei räumliche Regressionsmodelle vor, namentlich das „Simultaneous Autoregressive Modell“" und die „Geographisch Gewichtete Regression“, mit denen es möglich ist, räumliche Prozesse sowohl auf globalem als auch auf lokalem Maßstab zu beleuchten. Am Beispiel der Stadtregion Wien wird mit dieser Methodik untersucht, inwieweit ,harte“ Standortfaktoren wie z. B. Bodenpreise und Erreichbarkeiten die Zuzugsrate 20042006 beeinflussen. Darüber hinaus wird diskutiert, wie hoch deren Erklärungsgehalt vor dem Hintergrund neuerer postsuburbaner Prozesse innerhalb der Stadtregion ist.
\end{abstract}

Schlüsselwörter Suburbanisierung ·

Stadt-Umland-Wanderung · Wiener Umland (Österreich) ·

Räumliche Regression · Geographisch Gewichtete

Regression

Spatial Regression as a Useful Technique to Explore the Driving Forces of In-Migration in the Viennese Urban Region?
Abstract In metropolitan regions population developmen is a continuous and dynamic process and in matters of ana-
M. Helbich ( $₫) \cdot$ Dr. P. J. Görgl
Institut für Stadt- und Regionalforschung, Österreichische
Akademie der Wissenschaften,
Postgasse 7/4/2, 1010 Wien, Österreich
E-Mail: marco.helbich@oeaw.ac.at
Dr. P. J. Görgl
E-Mail: peter.goergl@oeaw.ac.at

lyzing it a huge variety of methodical approaches exists. This article introduces the simultaneous autoregressive model and the geographically weighted regression which can be seen as an innovative methodical enhancement of the usual global regression model. Using the urban region of Vienna as case study, the influence of "hard" location factors like land price, accessibilities etc. on in-migration for the period 2004-2006 is analyzed. The explanatory content of the approach as a whole and its additional value will be discussed conclusively, mainly with regard to newer, postsuburban processes in urban regions.

Keywords Suburbanization - Urban-to-rural migration · Driving forces · Vienna (Austria) $\cdot$ Spatial error model Geographically weighted regression

\section{Einleitung}

Stadtregionen unterliegen permanenten und kontinuierlichen Veränderungen, die stark von Stadt-Umland-Wanderungen der Bevölkerung geprägt sind. Gerade in rasch wachsenden Stadtumländern ist es von großem Interesse zu analysieren, welche Determinanten Zuwanderung generell bestimmen. Denn zum einen haben sich neue raumfunktionale Zusammenhänge zwischen Kernstädten und den Umlandgemeinden etabliert, die sich in eher polyzentralen, netzwerkartigen Siedlungsstrukturen niederschlagen und demzufolge nicht mehr ausschließlich auf die Kernstadt ausgerichtet sind (Sieverts/Koch/Stein/Steinbusch 2005). Zum anderen führte schon die „klassische“ Suburbanisierung der 1970er bis 1980er Jahre rasch zur planerischen Debatte um Zersiedelung des Umlandes, da das Wachstum nur in den seltensten Fällen als „geordnet“ bezeichnet werden kann. Im Hinblick auf die Knappheit verfügbaren Baulands bzw. 
eine immer weitere Ausdehnung der Stadtregionen und der zunehmenden internen funktionalen Vernetzung ist es von großer Relevanz zu analysieren, aus welchen Gründen Menschen einen Wohnstandort im Umland wählen. Denn kennt man die Motive der Wandernden genauer, lassen sich daraus (vielleicht) einmal auch Planungs- und Wachstumsstrategien ableiten, die weniger einer (auch den einzelnen Kommunen nur schwer vermittelbaren) ,top-down“-Ideologie entsprechen, als an den tatsächlichen Motiven der Akteure orientiert sind.

Im vorliegenden Beitrag wird in diesem Zusammenhang ein Blick auf das Wachstum in den Umlandgemeinden der österreichischen Stadtregion Wien geworfen. Dabei geht es in erster Linie nicht um die Frage, woher die Zuwanderer stammen und ob diese aus der Kernstadt in das Umland wandern oder ob es sich um Zuzüge von außerhalb der Stadtregion handelt, die sich ohne den Umweg über das Zentrum im Umland ansiedeln. Vielmehr sollen die Determinanten eingehender betrachtet werden, die bei jeder Standortentscheidung eine Rolle spielen (können). Auch wenn bekannt ist, dass die Wahl eines neuen Wohnstandortes eine individuell je einmalige Aneinanderreihung von Bedürfnissen, Wahrnehmungen, ökonomischen Ressourcen u. ä. ist, gibt es dennoch einige „Driving Forces“, die in nahezu allen Fällen eine gewichtige Rolle spielen.

Ein Blick in verschiedene Wohnmotivstudien oder die gegenwärtige Fachliteratur zur Entwicklung von Stadtregionen zeigt deutlich, dass Bodenpreise, Baulandverfügbarkeit, Erreichbarkeiten und ein naturräumlich attraktiveres Wohnumfeld mehr oder weniger unabhängig von der individuellen Situation entscheidende Kriterien dafür sind, an welchem Ort man sich schließlich niederlässt. Zahlreiche deutsche und österreichische Wohnmotivstudien kommen, trotz teils unterschiedlicher konzeptioneller Gestaltung und thematischer Ausrichtung des Untersuchungsdesigns, in diesem Bezug zu ähnlichen Ergebnissen (vgl. z. B. Blotevogel/Jeschke 2001; Institut für Medienforschung und Urbanistik 2002; Österreichisches Institut für Erwachsenenbildung 2004; Brake/Einacker/Mäding 2005; BMVBS/BBR 2007; Dittrich-Wesbuer/Osterhage 2008). Dabei handelt es sich eindeutig um „harte“ Standortfaktoren, die einerseits nicht gleichmäßig im Raum verteilt sind, wodurch eine spezifische stadtregionale Wertigkeit von Standorten entsteht (Bökemann 1999). Andererseits sind solche Faktoren quantifizierbar und eignen sich deshalb hervorragend für die statistische Analyse.

Dennoch zeichnet sich der hier vorgestellte Ansatz auch im internationalen Vergleich durch eine Methodik aus, die als „state of the art" der räumlichen Analyse bezeichnet werden kann. Zwar gibt es in Deutschland und Österreich durchaus Untersuchungen, die mittels deskriptiver und/oder multivariater Methoden räumliche Entwicklungen darstellen und erklären wollen (z. B. Giffinger/Kramar/Loibl 2001; Sie-
dentop/Kausch/Einig/Gössel 2003). Diese herkömmlichen Ansätze wurden in den letzten Jahren stetig weiterentwickelt und heute ist man, nicht zuletzt aufgrund der leichten Verfügbarkeit von Geodaten und der hohen Rechenleistungen von EDV-Systemen, in der Lage, komplexere Herangehensweisen, wie die hier angewendete Geographisch Gewichtete Regression (GWR) (vgl. Brunsdon/Fotheringham/Charlton 1996; Fotheringham/Charlton/Brunsdon 2002), - zur Beantwortung inhaltlicher als auch methodischer Fragen - zum Einsatz zu bringen. Durch die vorgestellte Methode lassen sich dazu nicht nur globale Aussagen treffen, sondern auch deren lokale, räumliche Spezifika abbilden, was für planerisch-raumanalytische Forschung immanent ist.

Allgemeines Ziel dieses Beitrags ist die Erklärung des räumlichen Verteilungsmusters von Zuzügen in Umlandgemeinden um die österreichische Bundeshauptstadt Wien anhand „harter“ Standortfaktoren. Im Detail werden folgende Fragen beantwortet: Welche „Driving Forces“ bestimmen die Zuzugsraten? Kommt es dabei zu räumlichen Variationen in deren Assoziationen bzw. verändert sich deren Beziehungsgeflecht im Raum?

Dieser Beitrag ist wie folgt strukturiert: Eingangs werden in Abschn. 2 das Untersuchungsgebiet sowie die herangezogenen Datensätze vorgestellt. Abschn. 3 präsentiert die angewandte Methodologie sowie die resultierenden Modellergebnisse. Geschlossen wird einerseits mit einer Diskussion der Empirie bzw. einer Einordnung derer in den theoretischen Kontext (Abschn. 4), andererseits werden in Abschn. 5 zukünftige Modellerweiterungen skizziert.

\section{Untersuchungsgebiet und Datengrundlage}

Wie für jede Stadtregion existieren auch für die österreichische Bundeshauptstadt Wien unterschiedliche Abgrenzungen. Für die vorliegende Analyse wurde eine bei „Praktikern“ der Raumordnungsabteilungen der drei Bundesländer Wien, Niederösterreich und Burgenland gängige Abgrenzung, die insgesamt 268 Gemeinden umfasst, gewählt. Diese im Vergleich zu älteren (vgl. z. B. Fuchs 1997) recht großzügige Variante hat den Vorteil, dass sie den österreichischen Teil der sogenannten CENTROPE Region (bestehend aus Teilräumen Österreichs, Ungarns, der Slowakei und Tschechiens) berücksichtigt und somit auch auf transnationale Entwicklungen reagiert. Denn nicht zuletzt der Verkehrsinfrastrukturausbau nach Tschechien oder in die Slowakei werden zu einem Entwicklungsschub in auch weiter entfernten Teilen des Wiener Umlands beitragen, die bislang eher durch Schrumpfung oder Stagnation aufgefallen sind.

Die Einwohnerzahl der gesamten Stadtregion beträgt im Jahr 2008 rund 2,7 Mio., wobei rund 900.000 auf das in Abb. 1 dargestellte Wiener Umland entfallen. Ab den 1960er Jahren fand im Wiener Umland Suburbanisierung 
Abb. 1 Verortung des Untersuchungsgebietes

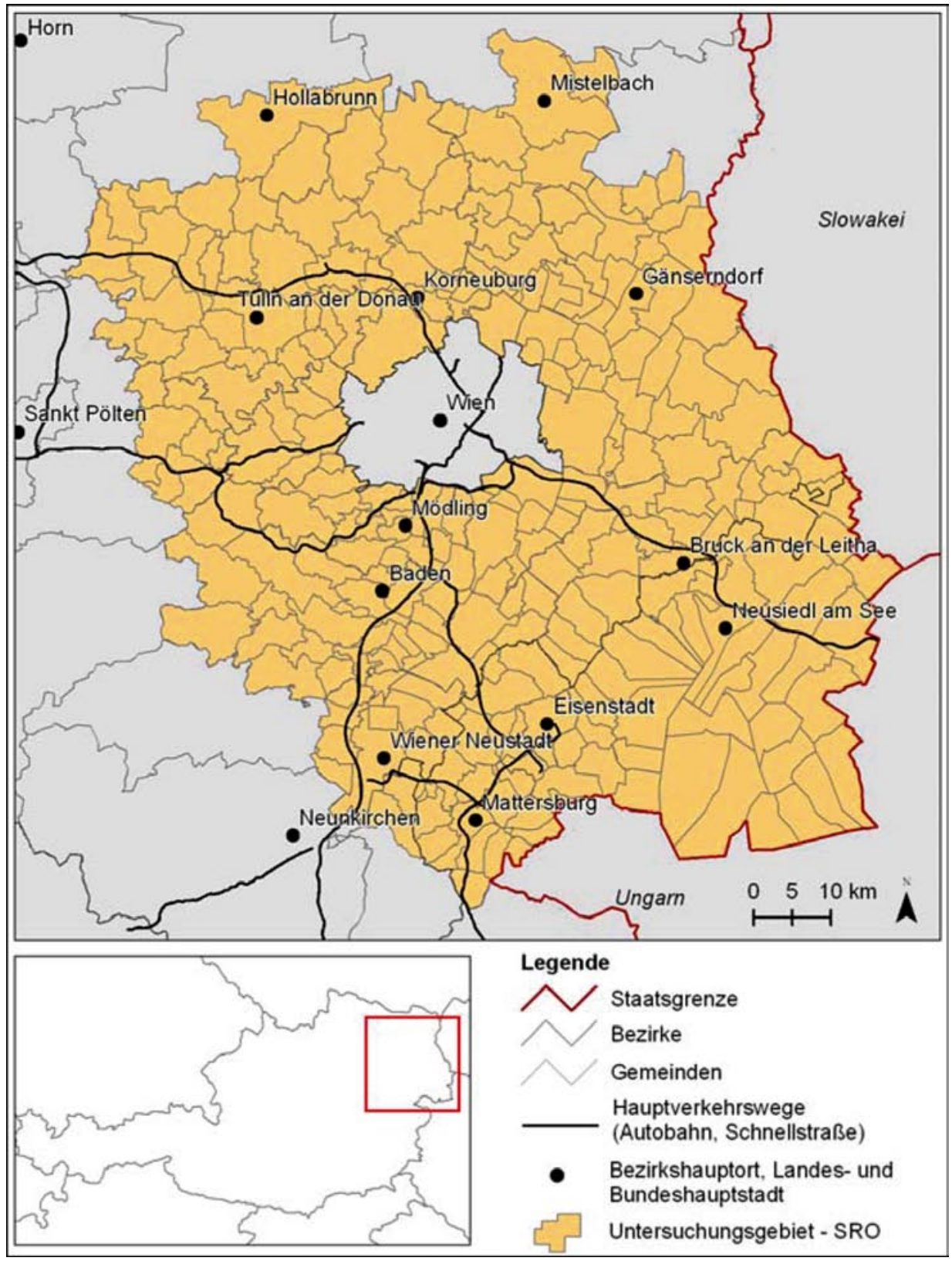

wstatt, wenngleich zunächst in viel geringerem Ausmaß als es etwa im Umland deutscher Großstädte wie z. B. in München oder Hamburg festzustellen war. Seit Mitte der 1970er Jahre haben sich einige Entwicklungsachsen herausgebildet, entlang derer zum Teil massive Bevölkerungszuwächse zu verzeichnen waren. Entlang der Südbahn in Richtung Mödling sowie Baden findet sich die stärkste Bevölkerungskonzentration. Im Nordwesten (Richtung Tulln und Korneuburg) oder Nordosten (Richtung Gänserndorf) sind weitere Entwicklungsachsen entstanden, die allerdings erst seit dem enormen Anstieg der Bodenpreise bei gleichzeitig schwindendem Baulandangebot im Süden an Bedeutung gewonnen haben. Generell ist die Bevölkerungszahl in der Stadtregion kontinuierlich angewachsen. Zwischen 2001 und 2006 nahm die Bevölkerung um rund 135.000 Personen zu, wobei der Großteil des Wachstums jedoch auf die Stadt Wien entfallen ist. Glaubt man Prognosen der Österreichischen Raumordnungskonferenz, soll diese Entwicklung auch in den kommenden Dekaden anhalten. Bis zum Jahr 2030 rechnet man mit einem Wachstum von bis zu 470.000 Menschen, die Hälfte davon in der Stadt Wien selbst und die andere Hälfte im Wesentlichen in den Umlandgemeinden der Stadtregion (Hanika/Biffl/Fassmann et al. 2004).

Die kleinste statistisch-territoriale Einheit, für die alle notwendigen Daten zur Verfügung stehen, ist die administrative Einheit der Gemeinde. Folglich wurden die Gemeinden zu den Merkmalsträgern, auf die in weiterer Folge Bezug genommen wird. Um fundierte Aussagen tätigen zu können, wurde 
Tab. 1 Datengrundlage

\begin{tabular}{|c|c|}
\hline Attribut & Quelle \\
\hline Zuzüge 2004, 2005, 2006 & POPREG Datenbank Statistik Austria \\
\hline $\begin{array}{l}\text { Bodenpreis für ein- und zweifamilienhaus-taugliche Baugrundstücke } \\
2006\end{array}$ & SRF TU Wien, GEWINN 7/8/2006 \\
\hline Landschaftliche Attraktivität & SRTM-DEM (NASA), Tele Atlas, IfGR Universität Wien \\
\hline $\begin{array}{l}\text { Erreichbarkeit Stadtzentrum Wien per Motorisierter Individualverkehr } \\
2005\end{array}$ & $\begin{array}{l}\text { Amt der NÖ Landesregierung, RU2, ERRAM-Erreichbarkeitsmodell } \\
2005\end{array}$ \\
\hline $\begin{array}{l}\text { Erreichbarkeit Zentraler Ort Stufe } 5 \text { Wien per Motorisierter } \\
\text { Individualverkehr } 2005\end{array}$ & $\begin{array}{l}\text { Beier/Friedwagner/Fürst et al. (2007), ERRAM-Erreichbarkeitsmo- } \\
\text { dell } 2005\end{array}$ \\
\hline $\begin{array}{l}\text { Erreichbarkeit Zentraler Ort Stufe } 3 \text { Wien per Motorisierter } \\
\text { Individualverkehr } 2005\end{array}$ & $\begin{array}{l}\text { Beier/Friedwagner/Fürst et al. (2007), ERRAM-Erreichbarkeitsmo- } \\
\text { dell } 2005\end{array}$ \\
\hline $\begin{array}{l}\text { Erreichbarkeit Stadtzentrum Wien per Öffentlicher } \\
\text { Personennahverkehr }\end{array}$ & Fahrplanauskunft des Verkehrsverbundes Ost Region (2009) \\
\hline
\end{tabular}

bei der Datenbeschaffung das Augenmerk auf Aktualität der Datenquellen gelegt, wobei der zeitlich kleinste gemeinsame Nenner auf das Jahr 2005 bzw. 2006 fiel. Tabelle 1 fasst die auf der administrativen Einheit der Gemeinde vorhandenen Daten sowie deren Quellen zusammen.

Um lokale Extrema bei Zuzügen auszugleichen, die beispielsweise infolge der Errichtung von Geschosswohnhäusern in kleineren Gemeinden auftreten können, wurde ein Mittelwert aus den Jahren 2004 bis 2006 gebildet. ${ }^{1}$ Da bei harten Standortfaktoren kurzfristige Schwankungen eher selten sind, wurde auf eine Ausdehnung des Beobachtungszeitraumes verzichtet. Ferner wird dies durch eine entsprechende Datenverfügbarkeit erschwert. Die Daten zum Bodenpreis 2006 lagen als Minimal- und Maximalwert je Gemeinde vor, wobei schlussendlich ein Mittelwert der Spannweite für die weitere Analyse herangezogen wurde. Etliche Datensätze, wie z. B. sämtliche Erreichbarkeitsberechnungen per motorisiertem Individualverkehr (MIV) oder der Index zur landschaftlichen Attraktivität, entstammen Modellierungen mittels eines Geographischen Informationssystems. Erstere wurden auf Basis des ERRAM-Modells (Erreichbarkeitsbasiertes Raster Raumanalysemodell) (vgl. Beier/Friedwagner/Fürst et al. 2007; Hemetsberger/Ortner 2008) berechnet. Dabei wurden die Fahrzeiten je Rasterzelle, auf Basis eines belasteten Straßengraphens zur morgendlichen Spitzenzeit, zu den entsprechenden Zielen (z. B. Zentrale Orte der Stufe 5) modelliert und im Anschluss anhand einer Mittelwertbildung je Gemeinde aggregiert. Hingegen wurde die Variable „landschaftliche Attraktivität“ approximativ als eine Funktion aus Reliefenergie sowie der Entfernung zu Wäldern und Gewässern mittels Map Algebra (Tomlin 1990) und Verfahren der multikriteriellen Evaluierung (Malczewski 1999) auf Basis eines Höhenmodells (DEM), eines Straßengraphens und eines Landnutzungsmodells operationalisiert. Für weitere Details sei auf Helbich (2009) verwiesen.

${ }^{1}$ Die mittlere Zuzugsrate in Prozent wurde wie folgt berechnet:

$$
\left(\frac{\sum \text { Zuzüge 2004-2006/3 }}{\sum \text { Bevölkerung 2004-2006/3 }}\right) * 100
$$

Abbildung 2 gibt einen groben Überblick über die räumlichen Verteilungsmuster sämtlicher herangezogenen Variablen, die als Push- bzw. Pull-Faktoren bei Zuzügen ins Umland wirksam werden können und sich in den erwähnten Studien zu Wohnstandortentscheidungen immer wieder finden ${ }^{2}$. Ohne im Detail auf die dargestellten Variablen eingehen zu wollen, lassen sich spezifische Verteilungsmuster erkennen. Beispielsweise sind Hotspots, im Sinne von Gemeinden mit hohen Zuzugsraten, im Süden, Nordwesten und Nordosten erkennbar. Analog dazu verhalten sich der Bodenpreis, landschaftliche Attraktivität und Erreichbarkeit. Alles in allem lässt sich festhalten, dass ein hoher Zuzug primär in Gemeinden erfolgt, die eine landschaftlich attraktive Lage haben, verkehrsinfrastrukturell gut erschlossen sind und folglich auch einen dementsprechend hohen Bodenpreis aufweisen. Welche dieser in Abb. 2 dargestellten Variablen bis zu welchem Grad tatsächlich einen Erklärungsbeitrag zu Zuzugsraten leistet, kann rein visuell kaum eruiert werden und bedarf folglich weiterer statistischer Analysen.

\section{Analysedesign und Ergebnisse}

Die Regressionsanalyse ist ein gängiges Verfahren zur Analyse von Beziehungen zwischen einer oder mehreren Variablen. Damit ist es einerseits möglich, auf die zugrunde liegenden Determinanten eines (räumlichen) Prozesses schließen zu können und andererseits Prognosen zu erstellen. Ziel ist es, die Effekte von harten Standortfaktoren auf die Bevölkerungssuburbanisierung zu ermitteln. Die Ableitung von Prognosen hinsichtlich zukünftiger Entwicklungstendenzen ist hingegen nicht Gegenstand dieser Analyse. Im Detail wird versucht, das räumliche Verteilungsmuster der Responsevariable mittlere Zuzugsrate 2004-2006 mittels der in Tab. 1 bzw. Abb. 2 angeführ-

\footnotetext{
${ }^{2}$ Abszissen- und Ordinatenwerte sämtlicher Karten repräsentieren die Rechts- und Hochwerte im MGI- (Militärgeographisches Institut) Bezugssystem.
} 

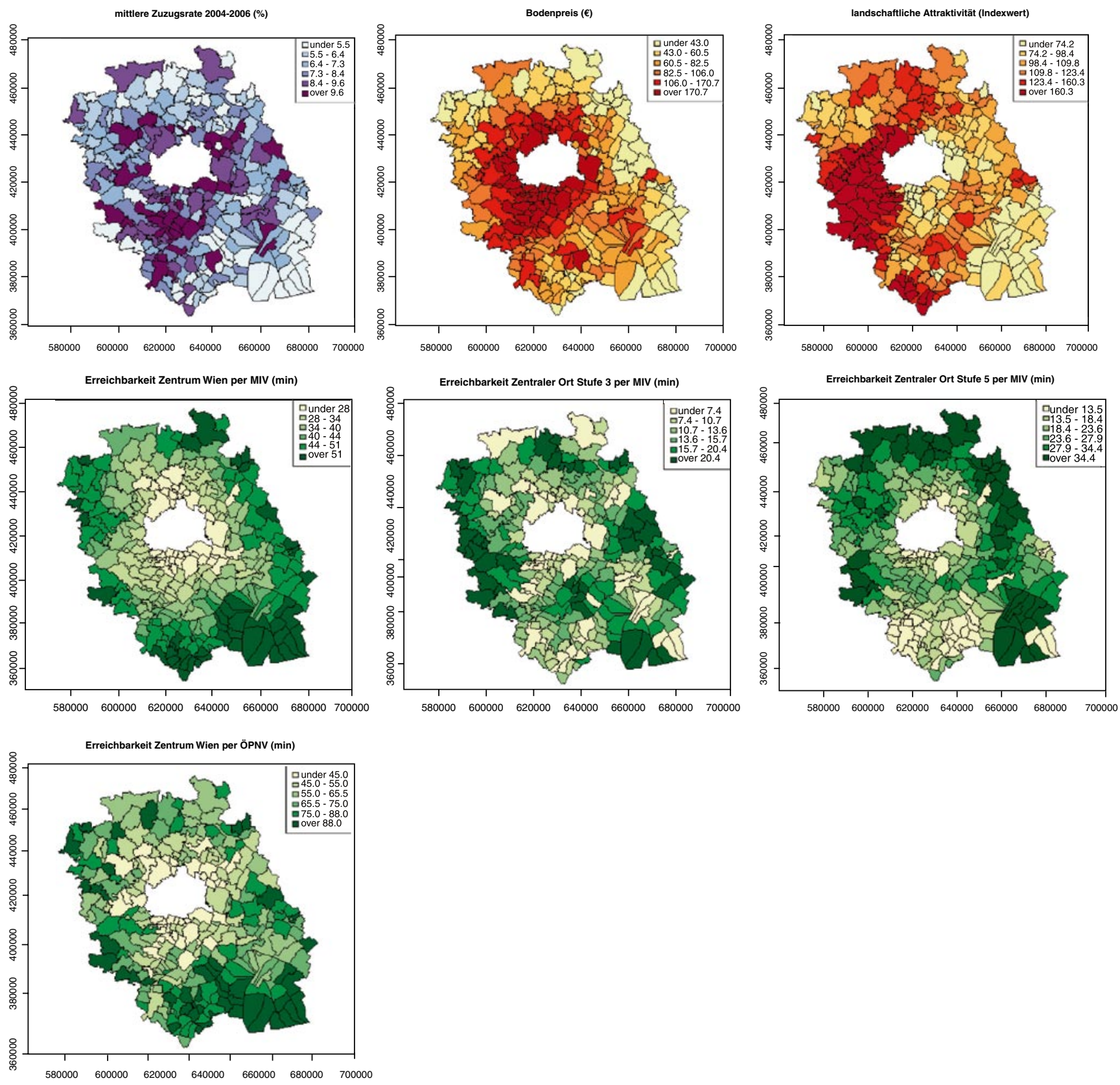

Abb. 2 Räumliche Verteilungsmuster des Variablensets

ten Prädiktoren zu erklären, um auf die für die Zuzugsrate essenziellen Driving Forces schließen zu können. Um adäquatere Verteilungseigenschaften (Normalverteilung) der Variablen mittlere Zuzugsrate und Bodenpreis zu erhalten, wurden diese logarithmiert. Im Folgenden wird das Forschungsdesign ergebnisorientiert erläutert, für methodische Details sei auf die entsprechenden Referenzen verwiesen (z. B. Anselin 1988, Fotheringham/Charlton/Brunsdon 2002).

In einem ersten Schritt wurde jenes Ordinary Least Square (OLS) Modell herausgefiltert, das nur mehr jene Variablen beinhaltet, die einen signifikanten Beitrag zur Erklärung der
Zuzugsrate leisten. Hierzu wurde eine schrittweise Modellselektion angewandt. Es zeigt sich - entgegen unserer a priori Annahme und perzeptiven Eindrücken - dass lediglich der Bodenpreis, die Erreichbarkeit Zentraler Ort Stufe 5 per MIV sowie die Erreichbarkeit des Stadtzentrums Wien per ÖPNV einen statistisch signifikanten Erklärungsbeitrag leisten (vgl. Tab. 2). Aufgrund des Vorhandenseins räumlicher Effekte - hier handelt es sich um räumliche Abhängigkeiten und Nicht-Stationarität - sämtlicher Eingangsvariablen konnte davon ausgegangen werden, dass zentrale Annahmen des Regressionsmodells verletzt werden. So ist beispielsweise die Responsevariable Zuzugsrate signifikant 
Tab. 2 Parameterschätzungen der globalen Regressionsmodelle

\begin{tabular}{|c|c|c|c|c|c|c|}
\hline & \multicolumn{3}{|l|}{ OLS } & \multicolumn{3}{|c|}{ Spatial Error Modell } \\
\hline & beta & Std. Fehler & t-Statistik & beta & Std. Fehler & z-Wert \\
\hline Intercept & 2,173 & 0,201 & $10,804^{* * *}$ & 2,116 & 0,220 & $9,598^{* * *}$ \\
\hline Bodenpreis (log) & 0,072 & 0,032 & $2,242^{*}$ & 0,079 & 0,036 & $2,178^{\dagger}$ \\
\hline $\begin{array}{l}\text { Erreichbarkeit MIV } \\
\text { ZO5 }\end{array}$ & $-0,003$ & 0,001 & $-2,125^{*}$ & $-0,002$ & 0,001 & $-1,518$ \\
\hline $\begin{array}{l}\text { Erreichbarkeit Wien } \\
\text { ÖPNV }\end{array}$ & $-0,007$ & 0,001 & $-7,098^{* * *}$ & $-0,006$ & 0,001 & $-6,421^{* * *}$ \\
\hline Lambda & - & - & - & $0,351^{* * *}$ & - & - \\
\hline F-Statistik & 49,$37 ; c$ & $=264 ; \mathrm{p}<0,0$ & & - & - & - \\
\hline Adj. $\mathrm{R}^{2}$ & 0,352 & - & - & - & - & - \\
\hline AIC & 26,283 & - & - & - & 9,505 & - \\
\hline
\end{tabular}

Signif. codes: $0^{(* * *)} ; 0,001^{(* *)} ; 0,01^{(*)} ; 0,05^{(\dagger}$

autokorreliert $^{3}$ und weist im Bereich des südlichen Umlands von Wien - auf Basis der lokalen Moran's I Statistik (Anselin 1995) - signifikante Hotspots auf (für Wien vgl. Helbich/ Leitner 2009). Dies bedeutet, dass die Zuzugsraten einer Gemeinde mit denen der räumlich benachbarten Gemeinden in Beziehung stehen und aufgrund dessen eine geographische Clusterung an territorialen Einheiten vorhanden ist. Demgemäß widerspricht dies der Unabhängigkeitsannahme der Beobachtungen. Diese Annahmenverletzung wurde durch eine Residuenanalyse des OLS-Modells bestätigt. Einerseits sind die Residuen signifikant räumlich korreliert ${ }^{4}$ und andererseits nicht normalverteilt ${ }^{5}$. Der Breusch-Pagan Test $^{6}$ bestätigt hingegen die Homoskedastizitätsannahme der Residuen. Eine Missachtung derartiger Annahmen des Modells verzerrt das Ergebnis und kann im Extremfall in falschen Schlussfolgerungen münden.

Dieser Umstand macht es erforderlich, eine Modellanpassung vorzunehmen, die diese räumliche Komponente explizit berücksichtigt. Hierzu bieten sich „Simultaneous Autoregressive Models“ (SAR) der räumlichen Ökonometrie an. Diese Modellgruppe beinhaltet etwa das Spatial Error Modell als auch das Spatial Lag Modell (Anselin 1988; Dormann/Mc Pherson/Araújo et al. 2007). Mittels der Lagrange Multiplier Teststatistik (LM) lässt sich nun eine statistische Aussage darüber treffen, ob der räumliche autoregressive Prozess in der Responsevariable oder aber in den Residuen modelliert werden soll. Ersteres entspricht dem Spatial Lag und zweites dem Spatial Error Modell. In diesem Falle verwies der robuste Lagrange Multiplier Test (RLM error) auf das Spatial Error Modell ${ }^{7}$. Im Anschluss an

\footnotetext{
${ }^{3}$ Moran's I: 0,363; $p<0,001$

${ }^{4}$ Moran's $I$ (error): 0,189; $p<0,001$

${ }^{5}$ Jarque-Bera Test: 52,149; $\mathrm{df}=2 ; p<0,001$

${ }^{6}$ Breusch-Pagan Test: 1,$409 ; \mathrm{df}=3 ; p=0,703$

7 Statistik: df: $p$-Wert:

LM error: $\quad 24,112 \quad 1 \quad 9,086 \mathrm{e}-07$

LM lag: $\quad 20,640 \quad 1 \quad 5,541 \mathrm{e}-06$

RLM error: $3,665 \quad 1 \quad 0,056$

RLM lag: $\quad 0,193 \quad 1 \quad 0,661$

SARMA: $\quad 24,305 \quad 2 \quad 5,274 \mathrm{e}-06$
}

diese modellstrukturelle Reformulierung wurde dieses abermals geschätzt und die Ergebnisse jenen des OLS-Modells in Tab. 2 gegenübergestellt. Formale Details zum Spatial Error Modell sind der Fußnote zu entnehmen ${ }^{8}$

Obwohl die Annahmen für eine OLS-Schätzung nicht gegeben waren, weisen beide Modelle im Großen und Ganzen nur geringe Unterschiede in den Parameterschätzungen auf. Jedoch erhöht sich der Erklärungsgehalt des räumlichen Modells erheblich. Beim OLS-Modell liegt das korrigierte Bestimmtheitsmaß (adj. $\mathrm{R}^{2}$ ) bei 0,35 und bietet einen nur mittelmäßigen Erklärungsgehalt. Der Wert des Akaike Informationskriteriums (AIC) liegt bei rund 26. Dieser Wert wird nun zur Modellevaluierung gegenüber dem Spatial Error Modell herangezogen. Die Reduktion dessen von 26 auf 9 verweist dabei auf eine deutlich bessere Performance des räumlichen Modells. Die Annahme von Varianzhomogenität der Residuen bestätigt sich auch im Spatial Error Modell' .

Betrachtet man nun jene Variablen, die signifikant zur Erklärung der Zuzugsrate beitragen, erkennt man, dass der im OLS-Modell noch signifikante Prädiktor Erreichbarkeit Zentraler Ort Stufe 5 per MIV nicht mehr als statistisch essenziell deklariert wird. Die beiden anderen Prädiktoren leisten hingegen weiterhin einen signifikanten Erklärungsbeitrag, auch wenn sich das Signifikanzniveau beim Bodenpreis von 0,01 auf 0,05 verringert hat. Im Falle des Spatial Error Modells ist der Prädiktor Bodenpreis mit der Zuzugsrate positiv und die Erreichbarkeit des Stadtzentrums Wien per ÖPNV negativ assoziiert. Weiters ist die SAR-Komponente Lambda in der Lage, einen großen Anteil der räum-

\footnotetext{
${ }^{8}$ Formal besitzt das Spatial Error Modell folgende Notation: $\mathbf{Y}=\mathbf{X} \beta+\mu \quad \mu=\lambda \mathbf{W}+\varepsilon, \quad \varepsilon \sim \mathrm{N}\left(0, \sigma^{2} \mathrm{I}\right)$

wobei $\mathbf{Y}$ ein $\mathrm{n} \times 1$ dimensionierter Vektor der abhängigen Variable, $\mathbf{X}$ ein $\mathrm{n} \times \mathrm{k}$ dimensionierte Matrix der $\mathrm{k}$ Prädiktoren, $\beta$ der Regressionskoeffizient, $\lambda$ ein Parameter der die räumliche Autokorrelation fasst, $\mathbf{W}$ die reihenstandardisierte Gewichtungsmatrix (die die Nachbarschaftsbeziehungen zwischen den Gemeinden mimt) und $\varepsilon$ der normalverteilte Fehler mit $\mathrm{N}\left(0, \sigma^{2} \mathrm{I}\right)$ ist.

${ }^{9}$ Breusch-Pagan Test für räumliche Modelle: 1,$660 ; \mathrm{df}=3 ; p=0,646$
} 
lichen Korrelation aufzunehmen, und ist ebenso höchst signifikant.

Interpretativ lassen obige Ergebnisse den Schluss zu, dass der Bodenpreis mit der Zuzugsrate in einer positiven Beziehung steht. Mit anderen Worten: Ein Anstieg der Zuzugsrate induziert einen Anstieg des Bodenpreises. Gegenteiliges trifft für die Variable Erreichbarkeit des Stadtzentrums Wien per ÖPNV zu. Die negative Assoziation mit der Responsevariable bedeutet, dass im Modell eine „Minderung“ der ÖPNV-Erreichbarkeit (im Sinne der Reduktion der Fahrzeiten) einen Anstieg der Zuzugsrate bedeutet. Dies heißt, dass längere Fahrzeiten zum Zentrum sich innerhalb der Stadtregion negativ auf die jeweilige Zuzugsrate auswirken.

Ein klarer Nachteil obiger globaler Modelle ist deren Annahme, dass im Untersuchungsgebiet keine geographisch variierenden Beziehungen in der Modellstruktur vorliegen. Die Resultate stellen somit nur über das gesamte Untersuchungsgebiet hinweg gemittelte Werte bzw. Beziehungen zwischen den Variablen dar, und lokale Besonderheiten bleiben demzufolge unberücksichtigt (Fotheringham/Charlton/Brunsdon 2002). Eine Methode zur Modellierung räumlicher Nicht-Stationarität ist die Geographically Weighted Regression (GWR) (Brunsdon/Fotheringham/Charlton 1996). Dabei handelt es sich um eine lokale Erweiterung des traditionellen globalen OLS-Regressionsansatzes, mittels dem nun räumlich unterschiedliche Beziehungen zwischen der Responsevariable und den Kovariaten modellierbar sind. Dies erfolgt über die Miteinbeziehung einer distanzabhängigen Gewichtung, bei der räumlich naheliegende Punkte stärker in die Berechnungen einfließen als weiter entfernte (Fotheringham/Charlton/Brunsdon 2002). Umgemünzt auf diese Analyse bedeutet das, dass für jede Gemeinde eine separate Regressionsanalyse durchgeführt wird und bei der Parameterschätzung zusätzlich eine gewisse Anzahl an Nachbargemeinden gewichtet miteinbezogen wird. Als Ergebnis erhält man lokale Varianten der Regressionsparameter, die nun kartographisch visualisierbar sind und präzisere Einblicke in das Beziehungsgeflecht zwischen den Determinanten erlauben. Das geschätzte GWR-Modell ist der Fußnote zu entnehmen ${ }^{10}$.

Im Vergleich zu den globalen Modellen in Tab. 2 lassen die GWR-Ergebnisse in Abb. 3 deutliche und signifikante räumliche Variationen in den Parameterschätzungen (F(3)-

\footnotetext{
${ }^{10}$ Hier wurde folgendes GWR-Modell geschätzt:

$\mathrm{y}_{\left(\mathrm{u}_{\mathrm{i}}, \mathrm{v}_{\mathrm{i}}\right)}=\beta_{0\left(\mathrm{u}_{\mathrm{i}}, \mathrm{v}_{\mathrm{i}}\right)}+\beta_{1\left(\mathrm{u}_{\mathrm{i}}, \mathrm{v}_{\mathrm{i}}\right)} \mathrm{x}_{1\left(\mathrm{u}_{\mathrm{i}}, \mathrm{v}_{\mathrm{i}}\right)}+\beta_{2\left(\mathrm{u}_{\mathrm{i}}, \mathrm{v}_{\mathrm{i}}\right)} \mathrm{x}_{2\left(\mathrm{u}_{\mathrm{i}}, \mathrm{v}_{\mathrm{i}}\right)}+\varepsilon_{\left(\mathrm{u}_{\mathrm{i}}, \mathrm{v}_{\mathrm{i}}\right)}$

wobei $\mathrm{y}_{\left(\mathrm{u}_{\mathrm{i}}, \mathrm{v}_{\mathrm{i}}\right)}$ die abhängige Variable Zuzugsrate, $\mathrm{x}_{1}$ der Prädiktor Bodenpreis, $\mathrm{X}_{2}$ der Prädiktor Erreichbarkeit des Stadtzentrums Wien per ÖPNV, $\left(\mathrm{u}_{\mathrm{i}}, \mathrm{v}_{\mathrm{i}}\right)$ die Koordinaten der Gemeindezentroide repräsentieren, $\beta_{\mathrm{k}\left(\mathrm{u}_{\mathrm{i}}, \mathrm{v}_{\mathrm{i}}\right)}$ eine biquadratische Funktion $\beta_{\mathrm{k}(\mathrm{u}, \mathrm{v})}$ mit konstanter Bandweite an der Position $\mathrm{i}$ und $\varepsilon$ der Fehler ist.
}

Test nach Leung/Mei/Zhang 2000) erkennen ${ }^{11}$, bei denen sich selbst mancherorts das Vorzeichen der Koeffizienten umkehrt. So etwa beim Prädiktor Bodenpreis, der beispielsweise in den nordöstlich an die Kernstadt angrenzenden Gemeinden einen negativen Beitrag bzw. Einfluss auf die Zuzugsrate ausübt, hingegen in den burgenländischen Gemeinden im Südosten des Untersuchungsgebietes einen positiven. Allgemein zeigt sich eine gewisse Distanzabhängigkeit dieser Determinante. Mit ansteigender Entfernung zur Stadtgrenze Wien steigt der Koeffizient kontinuierlich an und steht $\mathrm{ab}$ rund 45 Kilometer in positiver Beziehung mit der Zuzugsrate.

Ähnliches lässt sich für den zweiten Prädiktor Erreichbarkeit des Stadtzentrums Wien per ÖPNV feststellen. Im globalen räumlichen Modell nimmt der Regressionskoeffizient einen einheitlichen Wert von $-0,006$ an. Lokal betrachtet bewegt sich dieser in einer kleinen Anzahl an Gemeinden (z. B. Wolfsthal, Berg) in einem Bereich um Null herum und variiert im Rest des Untersuchungsgebietes im Bereich zwischen $-0,02$ und $-0,14$. Die geringsten Koeffizienten werden nordöstlich von Wien erreicht, wobei das Minimum in der stadtnahen Gemeinde Gerasdorf bei Wien erzielt wird.

Eine ähnliche Variation lässt sich auch für die Modellgüte, gemessen mittels lokalem Bestimmtheitsmaß $\left(\mathrm{R}^{2}\right)$, festhalten. In nördlichen bzw. nordöstlichen und südöstlichen Teilen nahe der Außengrenze des Untersuchungsgebietes weist das Modell lokale $\mathrm{R}^{2}$-Werte von 0,6 bis 0,9 auf. In anderen Teilen, insbesondere im Westen des Untersuchungsgebietes, lässt die Modellgüte mit Werten von 0,1 wiederum sehr zu wünschen übrig, was bedeutet, dass die Zuzugsrate in diesen Bereichen mit den beiden harten Standortfaktoren nur sehr bedingt erklärbar ist und weitere Determinanten zur Erklärung dieses Prozesses vonnöten sind. Zumeist aber werden lokale $\mathrm{R}^{2}$-Werte zwischen 0,2 und 0,4 erzielt. Alles in allem bescheinigt ein Test mittels ANOVA (Brunsdon/Fotheringham/Charlton 1999) dem GWR-Modell gegenüber dem OLS-Modell eine signifikant bessere Modellanpassung ${ }^{12}$. Sämtliche obige Berechnungen wurden in der Open Source Software R (R Development Core Team 2008) mittels dem spdep (Bivand 2008) und spgwr (Bivand/Yu 2008) Paket durchgeführt.

\section{Zusammenfassung und Bewertung der Ergebnisse}

In Bezug auf die Zuzugsraten in die Wiener Umlandgemeinden hat sich gezeigt, dass aus der Vielzahl der harten Standortfaktoren (vgl. Tab. 1), die als Ausgangsbasis für die Schätzungen der globalen Modelle dienten, lediglich die

\footnotetext{
${ }^{11} \mathrm{~F}(3)$-Test nach Leung/Mei/Zhang (2000) ergibt bei allen Prädiktoren einen $p<0,001$

${ }^{12}$ ANOVA: $F=2,726$; df1 =148,286; df2=244,826; $p=1,680 \mathrm{e}-12$
} 
Intercept

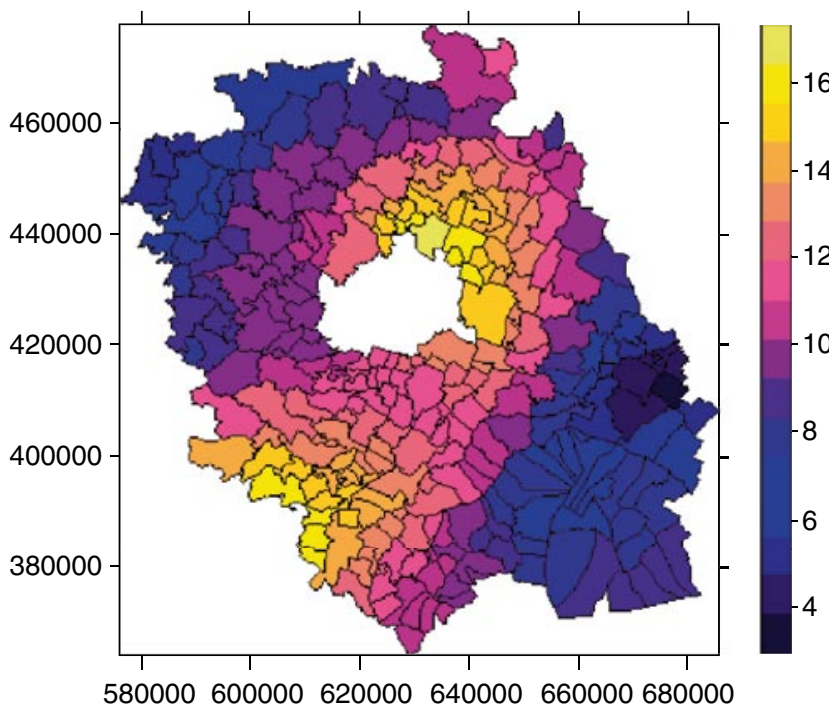

Erreichbarkeit ÖPNV Zentrum wien

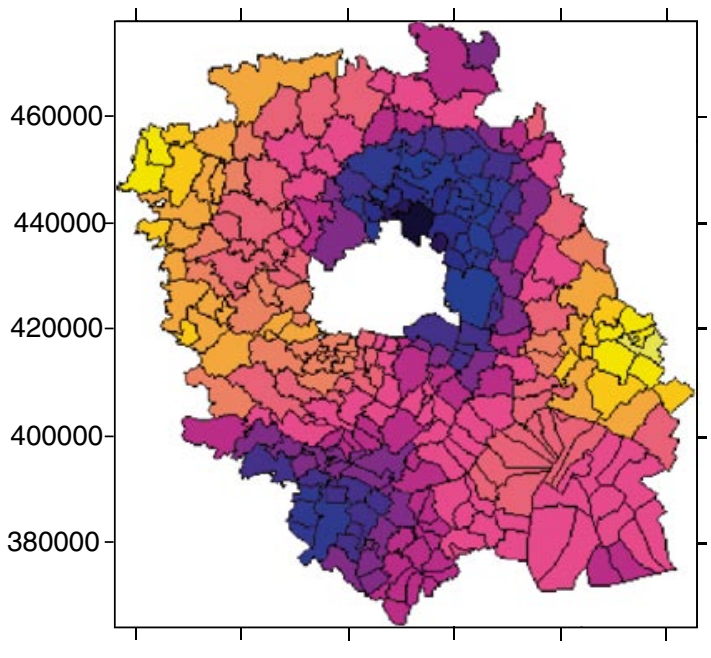

580000600000620000640000660000680000
Bodenpreis

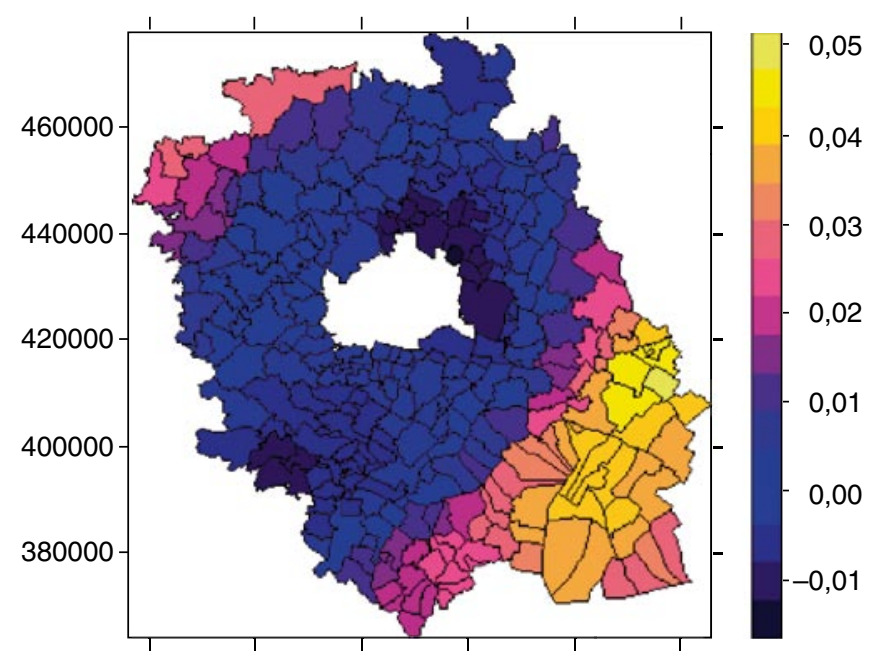

580000600000620000640000660000680000

Abb. 3 Parameterschätzungen des GWR-Modells

beiden Variablen Bodenpreis und ÖPNV-Erreichbarkeit des Wiener Zentrums einen statistisch relevanten Erklärungswert besitzen. Diese beiden im Spatial Error Modell signifikanten Prädiktoren (vgl. Tab. 2), wurden anschließend mittels Geographisch Gewichteter Regression im Detail analysiert, um eventuelle lokale Besonderheiten in deren räumlicher Variation der Parameterschätzungen (vgl. Abb. 3) aufzuzeigen.

Es können an dieser Stelle lediglich Vermutungen angestellt werden, weshalb die Pkw-Erreichbarkeit der Kernstadt in der Regression nicht inkludiert wurde, wo doch diese Einflussgröße in der Realität zweifelsohne eine enorme Wichtigkeit besitzt. Dieses Ergebnis gestattet aber nicht den Schluss, dass den real handelnden Akteuren die Erreichbarkeiten per ÖPNV „wichtiger“ sind. Es lässt sich ein (sta- tistischer) Zusammenhang dahingehend vermuten, dass die intraregionalen Pkw-Erreichbarkeitswerte weniger stark differieren als es bei der ÖPNV-Erreichbarkeit der Fall ist und ihnen infolgedessen innerhalb des Modells ein weniger starker Einfluss auf die Zuzugsraten nachzuweisen ist. So sind sowohl die Zuzüge als auch die Anbindungen der Gemeinden eher an den radialen Hauptverkehrsachsen orientiert und weniger an den periurbanen Zwischenräumen. Abseits der Achsenverläufe ergeben sich eklatant höhere Fahrzeiten. Beispielsweise ist das Stadtzentrum Wiens von der verkehrsmäßig gut angebundenen Bezirkshauptstadt Bruck an der Leitha in 46 Minuten erreichbar. Vergleicht man dies mit der Fahrzeit, die nötig ist, um von der zwischen den Verkehrsachsen gelegenen Gemeinde Scharndorf (einer von 
der administrativen Grenze Wiens in etwa gleich weit entfernten Gemeinde) in das Zentrum zu gelangen, so ist ein Anstieg auf rund 80 Minuten feststellbar. Diese Differenz ist beim motorisierten Individualverkehr deutlich schwächer ausgeprägt, da in Bezug auf das Straßennetz die räumliche Abgeschiedenheit von Standorten deutlich geringer zu Buche schlägt. Um beim obigen Beispiel zu bleiben, sind dies 32 Minuten von Bruck an der Leitha, das an der Hauptverkehrsachse liegt, und 36 Minuten von der Gemeinde Scharndorf aus.

Generell jedoch entspricht es durchaus alltagspraktischen Entscheidungs- und Bewertungsmustern, dass auch im vorgestellten Modell landschaftlicher Attraktivität und den Erreichbarkeiten Zentraler Orte unterer Stufen zugunsten der Erreichbarkeit des Zentrums und der Bodenpreise keine essenzielle Bedeutung beigemessen wird. Unabhängig von der Modellgüte und der damit einhergehenden Frage nach dem generellen Erklärungswert einzelner Variablen, lassen sich aus den Ergebnissen des GWR-Modells einige interessante Strukturen und Trends herauslesen, die etwas über die Entwicklung in der Wiener Stadtregion aussagen und abschließend diskutiert werden.

Betrachten wir einleitend die Determinante Bodenpreis. Zunächst zeigt sich, dass diese Variable in vielen Teilen des Umlands positiv auf die Zuzugsrate wirkt. Dahinter verbirgt sich eine auf den ersten Blick scheinbar paradoxe Erkenntnis, und zwar, dass Menschen dorthin ziehen, wo es teurer ist. Allerdings ist ein höherer Bodenpreis für ein- und zweifamilienhaustaugliche Baugrundstücke (gleiches gilt für Mietwohnungen) aber durchaus Ausdruck spezifischer Standortqualitäten, dabei kann es sich um eine besondere landschaftliche Gunstlage handeln wie um eine infrastrukturell sehr gut ausgestattete Gemeinde mit sehr guten Erreichbarkeitsverhältnissen. Es zeigt sich, dass in den peripheren Lagen der Stadtregion - sehr deutlich vor allem im südöstlichen Burgenland und im nordwestlichen Niederösterreich - der Einfluss des Bodenpreises auf die Zuzugsraten höher ist als in den wiennahen Teilgebieten, besonders entlang der „klassischen“ Suburbanisierungsachsen im Süden (Mödling-Baden) oder im westlich davon gelegenen Wienerwald, wo nur mehr vergleichsweise wenig Bauland ausgewiesen wird. Insofern spiegeln die unterschiedlichen lokalen Regressionskoeffizienten der Variable Bodenpreis auch unterschiedliche siedlungsstrukturelle Eigenheiten in der Stadtregion wider: Während in den peripheren Lagen der Einfamilienhausbau nach wie vor die dominierende Stellung innehat, muss man im stadtnahen Umland, entlang der älteren Suburbanisierungsachsen und in deren Zwischenräumen eine Vielzahl anderer Faktoren miteinbeziehen, die die Siedlungsstruktur beeinflussen, aber im vorliegenden Modell nicht berücksichtigt worden sind. Der hohe Anteil an Geschosswohnbauten ist hier ebenso zu nennen wie Weitergabe oder Vererbung bestehender Wohngebäude.
In Bezug auf die ÖPNV-Erreichbarkeit und ihre Wirkung auf die Zuzugsrate ist es logisch, wenn diese in den meisten Teilen der Stadtregion in einem negativen Zusammenhang stehen. Das bedeutet zunächst ganz allgemein: Je schlechter das Zentrum von einer Gemeinde aus zu erreichen ist, desto negativer wirkt sich das auf die Zuzugsraten aus. Doch ein Blick auf die entsprechende Karte in Abb. 3 zeigt, dass diese Beziehung nicht konstant und in deren Intensitätswerten nicht einheitlich verläuft. Als Bezugspunkt wurde die Erreichbarkeit der Wiener Oper gewählt. Aufgrund der Streckenführung im ÖPNV-Netz ergeben sich daher für die westlich und südlich gelegenen Umlandgemeinden Erreichbarkeitsvorteile, wohingegen die nördlichen Umlandgemeinden aufgrund öfter notwendigen Umsteigens u. ä. zu diesem Bezugspunkt zum Teil deutlich längere Fahrzeiten aufweisen. Dieser Umstand spiegelt sich auch in den diesbezüglich sehr unterschiedlichen Werten im näheren Umland Wiens wider $(-0,14$ im Norden und 0,06 im südlichen Umland). An dieser Stelle ließe sich diskutieren, ob der gewählte Bezugspunkt im Zentrum der Stadt tatsächlich gut gewählt wurde oder nicht. In Anbetracht der nahezu unüberblickbaren Vielzahl an Destinationen innerhalb einer Stadtregion wird eine solche Auswahl jedoch stets ein Kompromiss bleiben müssen.

Ebenso interessant scheinen im Hinblick auf die ÖPNVErreichbarkeit die recht nahe bei Null liegenden Werte in den peripheren Lagen an der West- und Ostgrenze der Stadtregion sowie in den Wienerwaldgemeinden im Westen zu sein. Gerade dort, wo nicht nur das Zentrum Wiens schlecht erreichbar ist, sondern der öffentliche Nahverkehr generell vergleichsweise schlecht ausgebaut ist, finden sich niedrige Werte. Dafür kann es eine einfache Erklärung geben: Wer sich für einen Wohnstandort in diesen Lagen entscheidet, ist nicht auf den öffentlichen Verkehr angewiesen, sondern eindeutig Pkw-orientiert. Darüber hinaus lässt sich vermuten, dass in diesen Lagen die Erreichbarkeit der Kernstadt bzw. ihres Zentrums generell eine untergeordnete Rolle spielt, weil die alltäglichen Arbeits- und Versorgungswege nicht dorthin führen. Insofern spiegelt diese Karte (vgl. Abb. 3) die mancherorts abnehmende Bedeutung der Variable Erreichbarkeit Zentrum wider.

Wirft man nun abschließend einen Blick auf die Güte des Modells an sich (lokales $\mathrm{R}^{2}$ ), also auf dessen Aussagekraft für eine Gemeinde unter Einbezug zahlreicher Nachbargemeinden, so zeigt sich ein insgesamt weniger erstaunliches Bild: Während in den peripheren Lagen der Erklärungswert an sich oftmals sehr hoch ist, nimmt dieser in den Teilgebieten im stadtnahen Umland stark ab. Daraus lässt sich nun der Schluss ziehen, dass in den Gemeinden der ersten Suburbanisierungsgeneration, also Teilgebieten, in denen gegenwärtig überdies auch eine Vielzahl postsuburbaner Dynamiken wirken (vgl. Görgl 2005; Görgl 2008), eindeutig andere wichtige Faktoren die Zuzugsrate beeinflussen. 
Die strategische Ausrichtung einer Gemeinde hinsichtlich der Aufnahme von zusätzlicher Wohnbevölkerung ist über Baulandausweisung und Einladung von institutionellen Wohnbauträgern wesentlich und erscheint im unmittelbaren Stadtumland sehr viel heterogener als im weit entfernten. Ebenso ist dies Ausdruck für den Sachverhalt, dass Wohnstandortentscheidungen eine äußerst komplexe Mixtur aus einer Vielzahl an individuell spezifischen Komponenten sind, in denen harte Standortfaktoren zwar eine wichtige Rolle spielen, aber kaum „alleinerklärend“ sind. So existieren in modernen Stadtregionen sowohl funktional als auch sozio-ökonomisch vielfältigste Verknüpfungen und Vernetzungen, die Ausdruck des polyzentrischen Lebens und Arbeitens sind und zu den heutigen Raumstrukturen geführt haben (Sieverts/Koch/Stein/Steinbusch 2005). Ein entsprechendes statistisches Analysemodell muss darauf mit einer differenzierten und überlegten Variablenauswahl reagieren.

\section{Ausblick}

Generell lässt sich festhalten, dass das Set an Ausgangsvariablen zukünftig breiter und noch differenzierter angelegt sein muss, als es in dieser Analyse der Fall gewesen ist. Beispielsweise müssen im Bereich Bodenpreise nicht nur Preise freier, ein- und zweifamilienhaustauglicher Grundstücke Eingang finden, sondern auch Variablen, die den Mietwohnungsmarkt widerspiegeln. Ähnliches gilt für die Frage nach Erreichbarkeiten. Wenngleich in dieser Analyse ein relativ breites Spektrum abgedeckt wurde, ist auch dieses in Anbetracht der niemals in ihrer Gesamtheit fassbaren Zahl an möglichen Wegekombinationen stets nur ein Kompromiss - wenn auch ein sehr logischer und nachvollziehbarer. Darüber hinaus ist es ebenso wichtig, die Palette an Einflussgrößen im Vorhinein zu erweitern und Ausstattungsmerkmale von Gemeinden sowie soziale Einflussgrößen (Kaufkraft, Bildung, Einkommen, Arbeitslosenzahlen etc.) in das Modell mit aufzunehmen.

Abgesehen von diesen kritischen Anmerkungen hat ein Ansatz wie das GWR-Modell aber Vorzüge, die andere Arten von Studien über Wohnstandortentscheidungen sehr gut ergänzen, indem es mittels einer innovativen Methodik in der Lage ist, die Einflussgrößen zu extrahieren, die sich auf Zuzugsraten am meisten auswirken, und dabei gleichfalls räumliche Variationen erfasst. Somit leistet dieser Ansatz einen sehr wichtigen Beitrag zum Verständnis der siedlungsstrukturellen Dynamik in Stadtregionen bzw. zum Verifizieren von Ergebnissen aus anders angelegten Studien zu diesem Thema.

Danksagung Dieser Artikel ist Teil der Grundlagenforschung zum Forschungsprojekt ,Strategien zur räumlichen Entwicklung der Ostregion“ (Laufzeit 2008-2010). Es wurde von der Planungsgemeinschaft
Ost (PGO), einem Zusammenschluss der österreichischen Bundesländer Burgenland, Niederösterreich und Wien, in Auftrag gegeben und wird unter anderem am Institut für Stadt- und Regionalforschung der Österreichischen Akademie der Wissenschaften unter der Leitung von Heinz Fassmann durchgeführt. In diesem Zusammenhang sind wir, stellvertretend für sämtliche Beteiligte, Herrn Walter Pozarek (PGO) zu herzlichem Dank verpflichtet. Weiters seien die konstruktiven Anmerkungen der Reviewer erwähnt.

\section{Literatur}

Anselin, L. (1988): Spatial Econometrics. Methods and Models. Dordrecht.

Anselin, L. (1995): Local Indicators of Spatial Association - LISA. In: Geographical Analysis 27, 2, 93-115.

Beier, R.; Friedwagner, A.; Fürst, B.; Gmeinhart, G.; Kurat, K.; Niko, W. (2007): Erreichbarkeitsverhältnisse in Österreich 2005. Modellrechnung für den ÖPNRV und den MIV. Wien = ÖROK Schriftenreihe, Nr. 174.

Bivand, R. mit Unterstützung von Anselin, L.; Assunçaõ, R.; Berke, O.; Bernat, A.; Carvalho, M.; Chun, Y.; Dormann, C.; Dray, S.; Halbersma, R.; Krainski, E.; Lewin-Koh, N.; Li, H.; Ma, J.; Millo, G.; Mueller, W.; Ono, H.; Peres-Neto, P.; Reder, M.; Tiefelsdorf, M.; Yu, D. (2008): Spdep: Spatial Dependence: Weighting Schemes, Statistics and Models. R package version 0.4-29. Online unter: $\mathrm{http}: / / w w w . R-p r o j e c t . o r g ~(l e t z t e r$ Zugriff am 21.05.2009).

Bivand, R.; Yu, D. (2008): Spgwr: Geographically weighted regression. R package version 0.5-4. Online unter: http://www.R-project. org (letzter Zugriff am 21.05.2009).

Blotevogel, H.; Jeschke, M. (2001): Determinanten der Stadt-UmlandWanderung im Raum Duisburg. Duisburg. = Diskussionspapier des Instituts für Geographie 4/2001.

Bökemann, D. (1999): Theorie der Raumplanung: Regionalwissenschaftliche Grundlagen für die Stadt-, Regional- und Landesplanung. München.

Brake, K.; Einacker, I.; Mäding, H. (2005): Kräfte, Prozesse, Akteure - zur Empirie der Zwischenstadt. Wuppertal.

Brunsdon, C.; Fotheringham, S.; Charlton, M. (1996): Geographically Weighted Regression: A Method for Exploring Spatial Nonstationarity. In: Geographical Analysis 28, 4, 281-298.

Brunsdon, C.; Fotheringham, S.; Charlton, M. (1999): Some Notes on Parametric Significance Tests for Geographically Weighted Regression. In: Journal of Regional Science 39, 3, 497-524.

Bundesministerium für Verkehr, Bau und Stadtentwicklung (BMVBS); Bundesamt für Bauwesen und Raumordnung (BBR) (Hrsg.) (2007): Akteure, Beweggründe, Triebkräfte der Suburbanisierung. Motive des Wegzugs - Einfluss der Verkehrsinfrastruktur auf Ansiedlungs- und Mobilitätsverhalten. Bonn. = BBR-onlinePublikation, 21/2007. Online unter: http://www.bbsr.bund.de/ cln_016/nn_340582/BBSR/DE/Veroeffentlichungen/BBSROnline/2007/DL_ON212007.html?_nnn=true (letzter Zugriff am 21.05.2009).

Dittrich-Wesbuer, A.; Osterhage, F. (2008): Wohnstandortentscheidungen in der Stadtregion: das Beispiel „Bergisches Land“. Dortmund. $=$ ILS Trends 2/2008. Online unter: http://www. ils-forschung.de/index.php?option $=$ com_content\&view $=$ article\&id=429\&itemid=107\&Itemid=107\&lang=de (letzter Zugriff am 15.09.2009).

Dormann, C.; Mc Pherson, J.; Araújo, M.; Bivand, R.; Bolliger, J.; Carl, G.; Davies, R.; Hirzel, A.; Jetz, W.; Kissling, W.; Kühn, I.; Ohlemüller, R.; Peres-Neto, P.; Reineking, B.; Schröder, B.; Schurr, F.; Wilson, R. (2007): Methods to Account for Spatial Autocorrelation in the Analysis of Species Distributional Data: A Review. In: Ecography 30, 5, 609-628. 
Fotheringham, S.; Charlton, M.; Brunsdon, C. (2002): Geographically Weighted Regression. The Analysis of Spatially Varying Relationships. Chichester.

Fuchs, I. (1997): Stadtregionen 1991 - Das Konzept. In: Statistische Nachrichten 2, 76-83.

Giffinger, R.; Kramar, H.; Loibl, W. (2001): Suburbanisierung in Österreich: ein steuerbarer Trend der Siedlungsentwicklung? In: Akademie für Raumforschung und Landesplanung (ARL) (Hrsg.): Stadt-Umland-Probleme und Entwicklung des großflächigen Einzelhandels in den Ländern Mittel- und Südosteuropas. Hannover, 25-49. = Arbeitsmaterial der ARL, Nr. 282.

Görgl, P. (2005): Structures postsuburbaines dans la région urbaine de Vienne. In: Revue Géographique de l'Est 45, 3-4, 133-144.

Görgl, P. (2008): Die Amerikanisierung der Wiener Suburbia? Der Wohnpark Fontana. Eine sozialgeographische Studie. Wiesbaden.

Hanika, A.; Biffl, G.; Fassmann, H.; Kytir, J.; Lebhart, G.; Marik, S.; Münz, R. (2004): ÖROK-Prognosen 2001-2031. Teil 1: Bevö1kerung und Arbeitskräfte nach Regionen und Bezirken. Wien. = ÖROK-Schriftenreihe, Nr. 166/I.

Helbich, M. (2009): Modellierung (post)suburbaner Prozesse am Fallbeispiel der Stadtregion Wien. Berlin u. a. = Beiträge zur europäischen Stadt- und Regionalforschung, Bd. 7.

Helbich, M.; Leitner, M. (2009): Spatial Analysis of the Urban-toRural Migration Determinants in the Viennese Metropolitan Area. A Transition from Suburbia to Postsuburbia? In: Applied Spatial Analysis and Policy 2, 237-260.

Hemetsberger, M.; Ortner, S. (2008): Erreichbarkeitsbasierte Raster Raumanalyse - Anwendungen in der Landesplanung. In: Schrenk, M.; Popovich, V.; Engelke, D.; Elisei, P. (Hrsg.): Mobility Nodes as Innovation Hubs. Proceedings of 13. International Conference on Urban Planning, Regional Development and Information Society. Schwechat, 255-263.
Institut für Medienforschung und Urbanistik (IMU) (2002): Raus aus der Stadt? Untersuchung der Motive von Fortzügen aus München in das Umland 1998-2000. Online unter: http://www.muenchen. de/Rathaus/plan/projekte/s_u_wanderung/41516/index.html (letzter Zugriff am 21.05.2009).

Leung, Y.; Mei, C.-L.; Zhang, W.-X. (2000): Statistical Tests for Spatial Nonstationarity Based on the Geographically Weighted Regression Model. In: Environment and Planning A 32, 1, 9-32.

Malczewski, J. (1999): GIS and Multicriteria Decision Analysis. New York.

Österreichisches Institut für Erwachsenenbildung (ÖIEB) (2004): Motivation und Zufriedenheit von Zuzüglern ins Wiener Umland. Zusammenfassung, Resümee, Empfehlungen. Online unter: http://www.oieb.at/download/OIEB-Zuzuegler_Zusammenfassung.pdf (letzter Zugriff am 21.05.2009).

R Development Core Team (2008): R: A Language and Environment for Statistical Computing. R Foundation for Statistical Computing. Wien. Online unter: http://www.R-project.org (letzter Zugriff am 21.05.2009).

Siedentop, S.; Kausch, S.; Einig, K.; Gössel, J. (2003): Siedlungsstrukturelle Veränderungen im Umland der Agglomerationsräume. Bonn. $=$ BBR-Forschungen, Nr.114.

Sieverts, T.; Koch, M.; Stein, U.; Steinbusch, M. (2005): Zwischenstadt - inzwischen Stadt? Entdecken, Begreifen, Verändern. Wuppertal.

Tomlin, D. (1990): Geographic Information Systems and Cartographic Modelling. Englewood Cliffs. 